\title{
Challenges to the theory of solar convection
}

\section{F. Cattaneo ${ }^{1}$}

${ }^{1}$ ASC/Flash Center, University of Chicago, 5640 S. Ellis Avenue, Chicago, Illinois 60637, USA email: cattaneo@flash.uchicago.edu

\begin{abstract}
Turbulent convection carries most of the solar luminosity from the stable radiative interior to the visible surface over a substantial fraction of the solar radius. The turbulent motions are highly nonlinear displaying activity on several spatial and temporal scales. The effects of these motions are to redistribute angular momentum, mix light elements, excite gravity waves and power magnetic activity. In this talk, I shall review some of the recent efforts, mostly based on numerical simulations, to model turbulent convection spanning multiple scale heights. I will discuss what we have learnt about the nature of convective transport, and what the relevant timescales are on which convection operates.
\end{abstract}

